\title{
Recovery of Isotopic Hotspots in Interplanetary Dust Particles by Focused Ion Beam Microscopy: Bridging the Gap between NanoSIMS and (S)TEM
}

\author{
G.A. Graham,* M. Bernas, ** Z.R, Dai,* J.P. Bradley,* J.B. Smith,* P.K. Weber, * H. Ishii,* and \\ I.D. Hutcheon,*. \\ * Institute of Geophysics \& Planetary Physics, Lawrence Livermore National Laboratory, \\ Livermore, CA 94551 \\ **Application Laboratory, FEI Company, Hillsboro, OR 97124
}

Interplanetary dust particles (IDPs) are a micron-sized $(\sim 5-50 \mu \mathrm{m})$ subset of meteoritic materials derived from comets or asteroids [1]. As IDPs represent some of the most primitive materials available, detailed study of their composition (mineralogy, chemistry and isotopes) gives fundamental insights into early solar system processes [1-3].

The next generation of ion microprobes are now capable of resolving isotopic anomalies of individual grains within IDPs [e.g. 2]. To fully benefit from such observations, it is important to be able to characterize the anomaly in terms of mineralogy or chemistry and its context in terms of the surrounding material within the IDP. In the example shown in Fig. 1, a hydrous IDP (L2047-D23) containing a nitrogen isotopic anomaly has been identified using the NanoSIMS 50 ion microprobe at LLNL [3].

Ultramicrotomy is the traditional method for preparing electron transparent sections of IDPs [4]. While it has been shown that the technique can be utilized to recover sections from IDPs previously prepared for nanoSIMS [2], it does not have the capability of guaranteeing the site-specific recovery of the isotopic anomaly. However, advances in ion beam milling techniques, particularly focused ion beam (FIB) microscopy, have revolutionized the preparation of TEM sections from bulk specimens [5].

An FEI Dual Beam 237 FIB/FESEM instrument was utilized to prepare a TEM section of material containing the anomaly. The region of interest within the IDP was initially coated with a film of $\mathrm{Pt}$ deposited in the FIB first using the electron beam and then using the ion beam. The Pt film or "strap" protects the specimen by reducing excessive superficial ion etching. The Ga+ ion beam operating at $5000 \mathrm{pA}$ current was then used to ablate material on either side of the protective "strap," extracted from the bulk material using an in-situ methodology [e.g. 5], and is subsequentially welded to a (half- cut) TEM grid using Pt. The section is then subjected to further milling at low currents (300pA down to $100 \mathrm{pA})$ and low voltage $(30 \mathrm{keV}$ down to $7 \mathrm{keV})$ to produce a TEM section $\sim 100 \mathrm{~nm}$ in thickness. The dual beam instrument used to prepare the section was fitted with a "flip-stage" that allowed the section to be oriented in a position that is suitable to SEM-STEM imaging. This allows real-time monitoring of the final stages of thinning and preliminary characterization of the final electron transparent section (Fig. 2a). Even at $30 \mathrm{keV}$ it is possible to texturally identify the presence of probable layer-silicates within the section. TEM confirmed the layer-silicates to be serpentine (basal lattice-fringe spacings of $\sim 0.7 \mathrm{~nm}$ ) (Fig. 2b-c).

The FIB recovery of anomalous grains from IDPs enables a new level of integrated analysis between the isotopic and mineralogical studies that will be a key driver in future astromaterials research. 


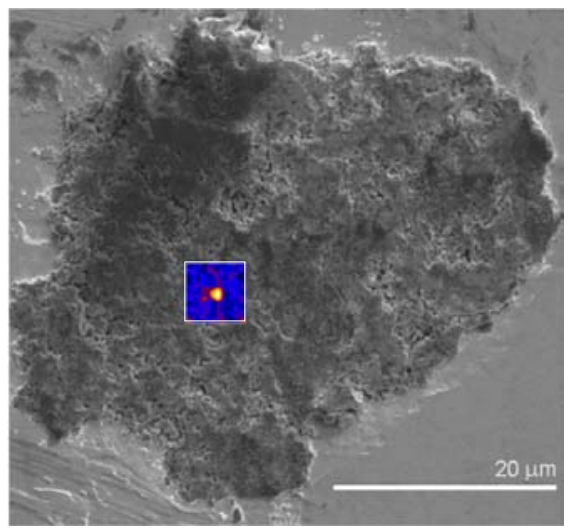

FIG. 1. A secondary electron image of IDP L2047-D23 pressed into gold foil. The insert shows the false color ${ }^{14} \mathrm{~N} /{ }^{15} \mathrm{~N}$ isotopic ratio image of the anomalous grain $(194 \pm 4(2 \sigma)$ compared to $272 \pm 2$ (solar value) for the bulk of the particle) identified within the IDP. The grain is approximately $800 \mathrm{~nm}$ in diameter (data taken from [3]).

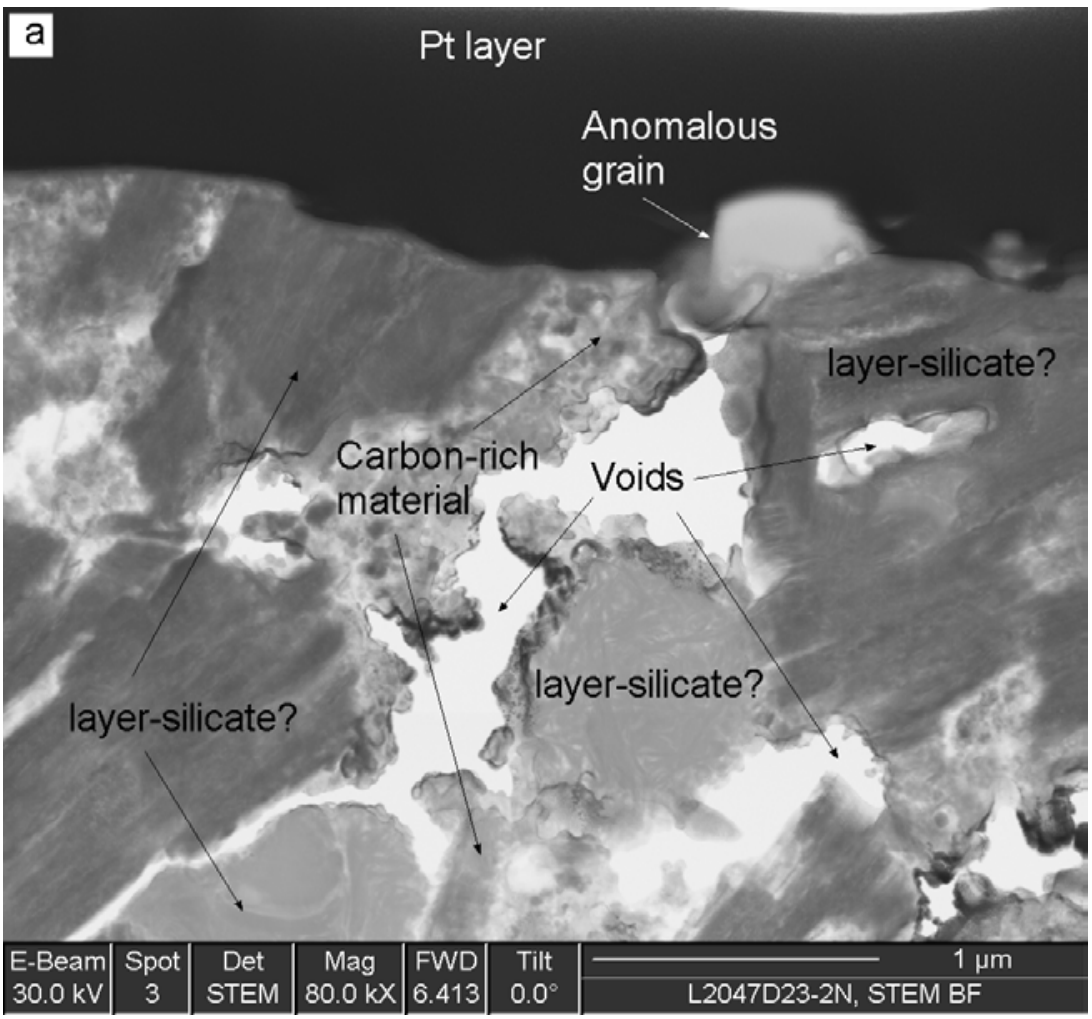

FIG. 2. (a) A 30keV SEM-STEM brightfield image of the extracted section after ion thinning to electron transparency. The fibrous phase is texturally indicative of a layer-silicate, e.g. serpentine or saponite. (b) Acquired lattice fringe image for the layer-silicate phase. (c) SAED pattern for the layer-silicate phase. (b\&c were acquired using CM300 FEGTEM)

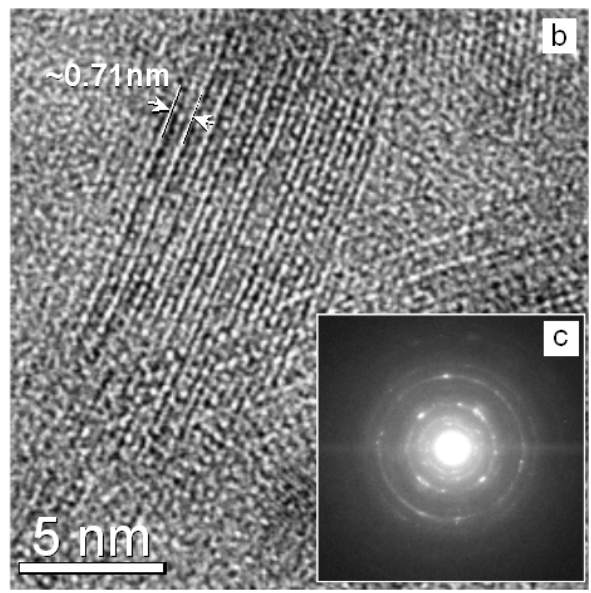

\section{References}

[1] J.P. Bradley, Treatise on Geochemistry, Vol. 1, p689, Elsevier, Amsterdam, 2003.

[2] S. Messenger et al., Science 300 (2000) 105.

[3] J.B. Smith et al., Lunar and Planetary Science XXXVI (2005) abstract \#1003.

[4] J.P. Bradley, Geochimica et Cosmochimica Acta 52 (1988) 889.

[5] L.A. Giannuzzi et al., Introduction to Focused Ion Beams, Springer, New York, 2005.

[6] This work was performed under the auspices of the U.S. Department of Energy, National Nuclear Security Administration by the University of California, Lawrence Livermore National Laboratory under contract No. W7405-Eng-48. The work is supported by NASA grants W-19894, NAG5-10632 and NAG5-10696. 